\title{
Thermal processes in ringholes
}

\author{
Pedro F. González-Díaz* \\ Colina de los Chopos, Instituto de Física Fundamental, Consejo Superior de Investigaciones Científicas, \\ Serrano 121, 28006 Madrid, Spain
}

(Received 11 June 2010; published 9 August 2010)

\begin{abstract}
Ringholes are space-time tunnelings connecting two asymptotically flat regions by means of a throat with the topology of a torus. This paper considers the processes of semiclassical thermal emission from ringholes and the accretion of dark energy onto them by adapting the results previously obtained for wormholes to toroidal topology. It is shown that at or near the throat the ringholes can be characterized as a mixture of two thermal sources, one at positive temperature and the other at negative temperature which, respectively, emit usual black body radiation and phantomlike radiation, leading after completion of thermal emission to two possible limiting situations, one similar to a wormhole in that it behaves just like a diverging lens, and the other similar to a black hole in that it behaves only as a converging lens. These two limiting situations, though described at rather diverging sizes, are also the final consequences from the accretion processes of quintessential and phantom vacuum energies. Finally, a brief discussion is added on the ringhole thermodynamics and its associated laws which are nothing but the extension to toroidal geometry from the thermodynamic laws which were recently established for wormholes.
\end{abstract}

DOI: 10.1103/PhysRevD.82.044016

\section{INTRODUCTION}

Similarly to how it once happened with black holes and more recently also with wormholes, the so-called ringholes [1] may now become a space-time construct pertaining to general relativity with remarkable pedagogical and even observational interest. Owing to their optical properties, which simultaneously corresponded partly to those of a divergent lens and partly to those of a convergent lens [1], ringholes actually might be expected to ultimately become some kind of mixture of black holes and wormholes [2,3], at least from an optical and a thermodynamical points of view. It is still unclear whether ringholes really exist in nature though the possible imprint that they would leave in the Universe appears as particularly distinctive in the form of two concentric bright rings [4], a signature which might have been already identified in a glowing double ring recently detected by the Hubble telescope [5].

Indeed a ringhole is nothing but a wormhole where the spherical throat symmetry has been replaced for the symmetry of a torus [1] (See Fig. 1). That more complicated topology allows any light ray or particle to follow an itinerary through the ringhole interior along which they may avoid finding any exotic or phantom matter but just ordinary matter, actually a safer way than that which can be followed through wormholes where the traveler inexorably becomes involved with matter having exotic properties, potentially incompatible with the very concept of life [1].

First of all, I would like to emphasize that there exists at least one nontrivial standpoint from whose perspective one might reach the rather tentative conclusion that, whereas the laws of quantum mechanics prohibited the existence of

*p.gonzalezdiaz@imaff.cfmac.csic.es
PACS numbers: 04.62.+v, 04.70.Dy

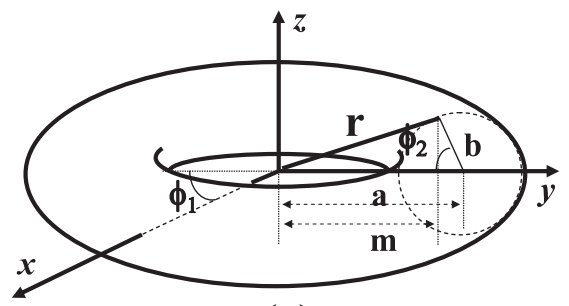

(a)

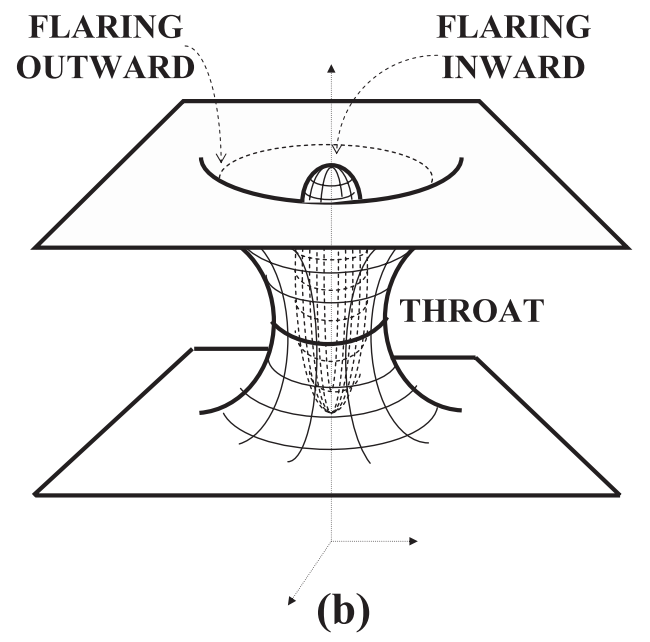

FIG. 1. (a) Geometrical parameters for the toroidal structure of the ringhole throat. (b) Pictorial representation of the space-time of a ringhole connecting two asymptotically flat regions belonging to the same universe, or to two different universes, as placed on a rectangular coordinate system. It satisfies the topology of a torus and, therefore, it can be embedded in a surface which partly flares outward, like a wormhole with spherical symmetry, and partly flares inward like a compact object made up of ordinary energy, such as a black hole or a neutron star. 
isolated wormholes, they in any event may become perfectly compatible with the presence of a ringhole, no matter whether it is isolated or not. This apparently unexpected result came about from the consideration of the so-called quantum interest conjecture of Ford and Roman [6], which follows from the quantum inequalities and according to which a positive energy pulse must always overcompensate any negative energy pulse by an amount that is a monotonically increasing function of the pulse separation. An isolated wormhole thermally radiating phantom energy at a negative temperature [7] seems to violate such a conjecture. However, in principle, from the double convergent/divergent character of the ringhole lens, the results of the present paper allow us to always accommodate the relative proportion of phantom to ordinary matter in a ringhole in such a way that, when thermally radiating (see Sec. III), the intensity of the positive internal energy pulses (where here and throughout the paper by internal energy we mean the quantity $p+\rho$ ) always overcompensated those containing phantom energy with $p+$ $\rho<0$.

In fact, it is a result of the present paper that due to the very structure of the throat of ringholes one should expect that the semiclassical thermal radiation processes spontaneously taking place in them actually become some sort of a mixture from thermal radiation being emitted from black bodies simultaneously radiating at positive and negative temperatures, a process which, as I have said above, at least could always satisfy the Ford and Roman conjecture [6] by adjusting the geometrical and topological parameters that define a ringhole (Fig. 1(a)) [4]. We also show in this paper that this thermal evaporation process of ringholes unavoidably leads to a remanent final space-time construct with a minimal size that is representable like some sort of either wormhole or black hole unable to radiate any more, provided that no accretion of dark or phantom energy [8] took place thereafter. If accretion continued the radiating processes, then we see that at the end of the day the same types of final spacetime constructs would be generated though now having the largest possible sizes, in fact overcoming that of the Universe and even diverging.

Keeping in mind a generalization of the Hayward $2+2$ formulation [7,9], this paper will deal with the semiclassical thermodynamical and thermal properties of ringholes and their connections with the allowed geometrical structure of these tunnels, always respecting the quantum interest conjecture (or its complementary version; see Sec. IV) in terms of a vacuum made up of positive (negative) internal energy. Placing then the ringhole in an accelerating Universe filled with dark energy [8], we will consider the processes related with the accretion of dark and phantom energy onto that ringhole and its subsequent evolution into a structure analogous to a wormhole or a black hole, or alike, from an optical and thermodynamical standpoints.

The contents of this paper can be outlined as follows: In Sec. II, we briefly review the geometry of a ringhole, with a special emphasis on the space-time metric and the optical properties of the corresponding geometrical construct as a converging and diverging lens. Section III contains an account of the semiclassical processes of thermal emission of positive- and exotic-energy radiation from a ringhole whose throat is characterized by both a positive and a negative temperature separated by angular horizons. Two limiting ringhole geometries are derived and discussed as the final stage of the evaporation processes with the smallest nonzero sizes. Section IV contains a detailed study of the dark energy accretion onto ringholes which is also performed starting from a perfect-fluid model and integrating the conservation laws for both the energy-momentum tensor and its projection on the four-velocity in the case of a nonstatic metric. The same two distinct types of limiting geometries as in the preceding section also reappear at the end of the accretion processes, this time having the largest possible sizes. I very briefly summarize in Sec. V where I add a short account of plausible thermodynamical laws of the ringholes and the concept of generalized entropy.

\section{THE RINGHOLE SPACETIME}

In this section, I shall very briefly review the characteristics of the space-time of a ringhole, particularly emphasizing its properties as a double (diverging and converging) optical lens. Using the customary ansatz for a tunneling topology in terms of a constant redshift function and the other familiar wormhole parameters [10], we can finally obtain for the four-dimensional space-time metric of a ringhole [1] a static metric which, in order to check the properties of a ringhole as an optical lens, can be written in the form [1]

$$
d s^{2}=-d t^{2}+\left(\frac{n}{r}\right)^{2} d \ell^{2}+m^{2} d \varphi_{1}^{2}+\left(\ell^{2}+b_{0}^{2}\right) d \varphi_{2}^{2},
$$

where (see Fig. 1(a)) $a$ and $b$ are the radius of the circumference generated by the circular axis of the torus and that of a torus section, respectively, with $a>b,-\infty \leq t \leq \infty$, $a-b \leq r \leq a+b$ and the angles $0 \leq \varphi_{1}, \varphi_{2} \leq 2 \pi$, with $-\infty<\ell<+\infty$ the proper radial distance of each transversal section of the torus, and

$$
\begin{gathered}
m=a-\left(\ell^{2}+b_{0}^{2}\right)^{1 / 2} \cos \varphi_{2}, \\
n=\left(\ell^{2}+b_{0}^{2}\right)^{1 / 2}-a \cos \varphi_{2}, \\
r=\sqrt{a^{2}+\ell^{2}+b_{0}^{2}-2\left(\ell^{2}+b_{0}^{2}\right)^{1 / 2} a \cos \varphi_{2}},
\end{gathered}
$$

in which $b_{0}$ is the throat radius. As $\ell$ increases from $-\infty$ to $0, b$ decreases monotonously from $+\infty$ to its minimum value $b_{0}$ at the throat radius, and as $\ell$ increases onward to $+\infty, b$ increases monotonously to $+\infty$ again. Now, for metric (1) to describe a traversable ringhole we must embed it in a three-dimensional Euclidean space at fixed time $t$ [10]. We should consider a three-geometry that 
would respect the topology of a torus and satisfy $a \geq b \leq$ $\ell$, so it will suffice to confining attention to the maximumand minimum-circumference slices, that is $\varphi_{2}=\pi, 0$, through it. In the first case, $r=m=n=a+b$, and

$$
d s^{2}=\frac{d r^{2}}{1-\frac{b_{0}^{2}}{b^{2}}}+r^{2} d \varphi_{1}^{2} .
$$

We visualize then this slice as removed from space-time (1) and embedded in the three-dimensional Euclidean space which is taken to be given in terms of cylindrical coordinates such as

$$
d s^{2}=d z^{2}+d r^{2}+r^{2} d \phi^{2}=\left[1+\left(\frac{d z}{d r}\right)^{2}\right] d r^{2}+r^{2} d \phi^{2},
$$

with $d z / d r=\left(b^{2} / b_{0}^{2}-1\right)^{-(1 / 2)}$ in order for the metric (4) to be the same as the previous one. This condition displays the way in which, provided we have fixed $a \geq b$, the function $b \equiv b(\ell)$ shapes the ringhole spatial geometry. In the case that we consider the minimum-circumference slice, $\varphi_{2}=0$, through the geometry of three-space at a fixed time, then $r=m=-n=a-b$, and the previous metric is obtained again, so that we always achieve the latter condition, no matter the choice of slice.

The requirement that ringholes be connectable to asymptotically flat space-time entails at the throat that the embedding surface flares outward for $2 \pi-\varphi_{2}^{c}>\varphi_{2}>$ $\varphi_{2}^{c}$, and flares inward for $-\varphi_{2}^{c}<\varphi_{2}<\varphi_{2}^{c}$, with $\varphi_{2}^{c}=$ $\arccos (b / a)$, which, respectively, satisfy the condition $d^{2} r / d z^{2}>0$ and $d^{2} r / d z^{2}<0$, at or near the throat. It follows [1] that one had to expect lensing effects to occur at or near the ringhole throat, that is to say, the mouths would act like a diverging lens for world lines along $2 \pi-$ $\varphi_{2}^{c}>\varphi_{2}>\varphi_{2}^{c}$, and like a converging lens for world lines along $-\varphi_{2}^{c}<\varphi_{2}<\varphi_{2}^{c}$. No lensing actions would therefore take place at the angular horizons placed at $\varphi_{2}=\varphi_{2}^{c}$ and $\varphi_{2}=2 \pi-\varphi_{2}^{c}$.

In fact, in the case of ringholes, instead of producing just a single flaring outward for light rays passing through the wormhole throat [10], this multiply connected topology, in addition to that flaring outward (diverging) effect, also produces a flaring inward (converging) effect [1] on the light rays that pass through its throat, in such a way that an observer on Earth would interpret light passing through the ringhole throat from a single luminous source as coming from two bright, glowing concentric rings, which form the distinctive peculiar pattern from ringholes [4]. That pattern cannot be generated by any other possible disturbing astronomical object other than the very implausible set of three luminous massive objects (let us say galaxies) which must be so perfectly aligned along the line of sight that its occurrence becomes extremely unlikely [5].

The reader interested in the physics and space-time structure of ringholes can find much more material in
Refs. $[1,11]$. However, we now add in what follows some new arguments to consolidate even further what was said in these references. First of all, I point out that if I enclose a ringhole and a wormhole in a box, one would expect the latter hole to undergo a phase transition whereas the phase of the former hole would remain unchanged. Hence one would also expect that ringholes are more stable than wormholes. On the other hand, to better understand what a ringhole space-time actually is it could appear useful as well to explain the possible relation of the ringhole to the so-called toroidal black holes [12]. Really, even though there exist theorems regarding topological censorship which are in apparent conflict with the collapse of matter to form a toroidal black hole, Shapiro et al. have shown [13] that the geometry of a temporarily toroidal event horizon is completely consistent with the alluded theorems. In particular, there is a theorem by Hawking which states [14] that the topology of a black hole must be spherical both in vacuum and in the presence of a positive cosmological constant, but if the metric is asymptotically anti-de Sitter, then it is possible to have a toroidal event horizon formed.

Now, similarly to the case of a Schwarzschild wormhole (that is, a Einstein-Rosen bridge [15]), at first sight, one could think of a ringhole as an asymptotically anti-de Sitter vacuum solution formed by one such toroidal black hole where the central singularity is replaced for a toroidal white hole. Such a space-time construct would nevertheless be equivalent to an asymptotically anti-de Sitter Einstein-Rosen bridge, and therefore become unstable and hence not traversable, preventing even light from making it through, as it ought to pinch immediately off at the so constructed throat [16]. The kind of ringhole we are dealing with is no longer a vacuum solution for negative cosmological constant, but it requires some proportion of ordinary matter and some proportion of exotic matter like what is used to construct a Thorne-Morris wormhole. Such a matter combination holds the throat open and actually makes the ringhole traversable and more stable than any wormholes.

\section{THERMAL EMISSION FROM RINGHOLES}

The ultimate physical reason why a wormhole radiates phantom energy at a negative temperature lies [7] in the feature that the surface embedding the wormhole spacetime flares outward at or near the throat, so that, unlike most gravitational systems made out of ordinary matter, such as stars or black holes, the wormhole throat really always behaves like an optical diverging lens under any circumstances. That is no longer the case with a ringhole where the optical properties that can be ascribed to its throat stem now from the feature that, depending on the value taken on the angle, $\varphi_{2}$ (formed up by the radius of the torus section, $b$, with that of the circumference generated by the circular axis of the torus, $a$, with $a>b$ ), see 
Fig. 1(a), the embedding surface flares either outward if $2 \pi-\varphi_{2}^{c}>\varphi_{2}>\varphi_{2}^{c}$, or inward when $-\varphi_{2}^{c}<\varphi_{2}<\varphi_{2}^{c}$, with $\varphi_{2}^{c}=\arccos (b / a)$.

Neither flaring nor lensing actions would therefore take place at the two existing angular horizons at $\varphi_{2}=\varphi_{2}^{c}$ and $\varphi_{2}=2 \pi-\varphi_{2}^{c}$. It appears then that it is most natural to expect from the very onset that, from the embedding surface sector defined by $2 \pi-\varphi_{2}^{c}>\varphi_{2}>\varphi_{2}^{c}$, ringholes would radiate phantom or exotic radiation at a negative temperature, and from the embedding surface sector $-\varphi_{2}^{c}<\varphi_{2}<\varphi_{2}^{c}$, separated from the previous one by means of the above two angular horizons, the same ringhole simultaneously radiated ordinary positive-energy particles at a positive temperature, both along thermally chaotic processes; the negative internal energy radiation always being overcompensated by the positive internal energy radiation in the case that the vacuum surrounding the ringhole be characterized by a positive-energy fluid, like quintessential dark energy is [17], or vice versa, the positive internal energy radiation always being overcompensated by the negative internal energy radiation when the surrounding vacuum is taken to be a fluid with negative internal energy, like phantom energy [18] (see next section).

We now derive the formulas for these temperatures and their dependence on the geometrical parameters defining the ringhole surface (Fig. 1(a)), working as usual in the semiclassical approximation where quantum radiation is propagated through the above considered classical spacetime. In order to obtain a convenient expression for the surface gravity of a ringhole, we have taken into account the $2+2$ formalism first advanced by Hayward for spherically space-times [10], where a generalized surface gravity is defined on the trapping horizon. Now, keeping in mind that formalism and taking into account the existence of the above mentioned $\varphi_{2}$-angular horizons, we can adapt the results of Ref. [7] to our toroidal geometry by introducing a suitable ansatz to describe the surface gravity on the trapping horizon of a ringhole. The surface gravity for the case of a ringhole can be then written as an extension to toroidal symmetry from the surface gravity in the spherically symmetric case that corresponds to a wormhole, derived in Ref. [7] by solving the actual equations of the general Hayward formalism, when applied to a wormhole. Using the expression for $\kappa$ of Refs. [7,10], we have then for ringholes

$$
\begin{aligned}
\kappa= & \frac{b_{0}^{2}}{2 b\left(a^{2}+b^{2}-2 a b \cos \varphi_{2}\right)}-2 \pi\left(a^{2}+b^{2}\right. \\
& \left.-2 a b \cos \varphi_{2}\right)^{1 / 2}\left(\epsilon-p_{r}\right),
\end{aligned}
$$

with $\epsilon$ the energy density and $p_{r}$ the radial pressure which depends on the angle $\varphi_{2}$, too. Their combination in Eq. (5) reads [1]

$$
\epsilon-p_{r} \simeq-\frac{c^{4} n^{2} b_{0}^{2}}{8 \pi G r^{3} b^{3}}\left(2+\frac{m a}{n b}+\frac{n b}{m a}\right) .
$$

In the interval $2 \pi-\varphi_{2}^{c}>\varphi_{2}>\varphi_{2}^{c}$ the combination $\epsilon-$ $p_{r}$ becomes negative [1] and hence $\kappa$ is definite positive. In the case $-\varphi_{2}^{c}<\varphi_{2}<\varphi_{2}^{c}$, then [1] $\epsilon-p_{r}>0$ and therefore the generalized surface gravity $\kappa$ becomes definite negative, after passing through $\epsilon-p_{r} \simeq 0$ on the two angular horizons.

Now, from the general expression for gravitational temperature $T_{G}$ (see, for example, Ref. [7])

$$
T_{G}=\frac{-\left.\kappa\right|_{b=b_{0}}}{2 \pi},
$$

it follows then that, as was to be expected for an absolute value of the radius, $a$ is sufficiently larger than that for radius $b$; i.e. for $\kappa \simeq-2 \pi \sqrt{a^{2}+b^{2}-2 a b \cos \varphi_{2}}\left(\epsilon-p_{r}\right)$ : (i) whereas the temperature on the embedding surface that flares outward is always negative, that on the flaring inward embedding surface is always positive and its absolute value is either larger or smaller than that for the negative temperature, depending on the nature of the surrounding vacuum, and (ii) in spite of that, because the involved negative-temperature system and the positive-temperature system can never come in contact in the present case (because the temperature vanishes at the two angular horizons), and hence the former system is not definitely hotter or colder than the latter one [19], at least at the first stages of a large- $a$ ringhole evaporation, the intensity and energy of the radiation pulses emitted at the positive temperature may well overcompensate those generated at a negative temperature or vice versa, so satisfying the generalized quantum interest conjecture (see the last two paragraphs of the next section).

It also follows that, as the above overall thermal process at combined positive- and negative-temperature emission progresses, the ringhole may be converted into either some sort of wormhole in that the surface embedding it would tend to only flare outward (that precisely happening when $a$ becomes exactly equal to $b$ [see Fig. 2(a)]), or some sort of black hole in that the embedding surface tended to just flare inward (this taking place when $a=-b$ [see Fig. 2(b)]). These limiting geometric ringhole configurations either can only continue emitting phantom radiation at a negative temperature as far as the usual quantum interest conjecture [6] is violated (see Sec. IV), such as it also happens with spherically symmetric wormholes, or can emit ordinary radiation at a positive temperature such as it happens with spherically symmetric black holes. It is in this sense that ringholes with these limiting geometries are equivalent either to a wormhole or to a black hole. There is still another aspect in which wormholes or black holes and the above limiting ringhole configurations are again equivalent. It is in that both types of tunneling (wormholes and limiting ringholes with $a=b$ ) and both 
types of compact space-time objects (black holes and limiting ringholes with $a=-b$ ) would all show the same gravitational signature on the sky when light coming to us from a luminous object is placed behind them, along the line of sight, i.e. a single glowing ring of the kind already considered by Shatskiy for single wormholes [20]. Unfortunately, such bright rings are not at all distinguishable from, e.g. Einstein rings generated by, e.g. galaxies, or that which is produced by stars with negative energy.

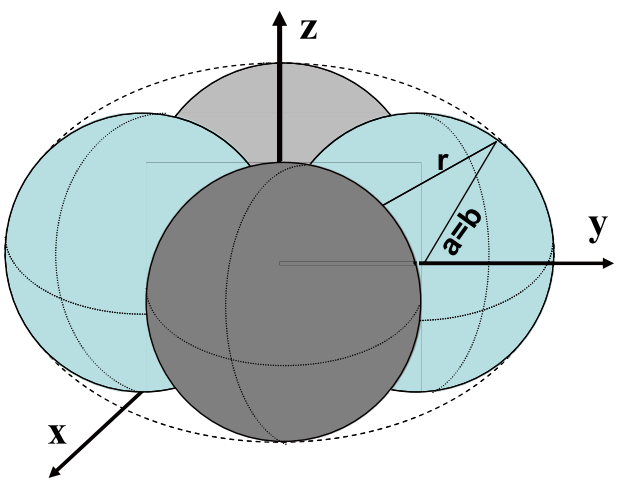

(A)

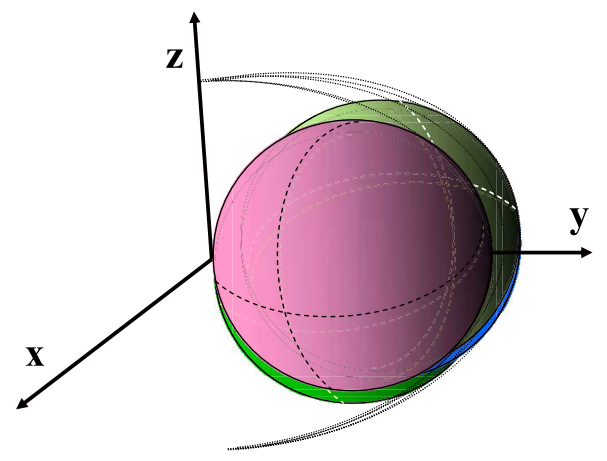

(B)

FIG. 2 (color online). Limiting ringholes in which $a^{2}=b^{2}$. We have then two possible final situations: (a) if $a=b$, then the process of thermal emission of positive- and negative radiation tends to that limiting geometry where all ordinary energy contributing the ringhole with $a>b$ is exhausted, leaving a geometry that is equivalent to that of a wormhole in that the surface embedding it flares outward but not inward, and is therefore made out of exotic, phantomlike energy characterized by a negative temperature; and (b) if $a=-b$, then the process of thermal emission tends to that limiting geometry where now all exotic energy contributing the ringhole with $|a|>|b|$ is exhausted, leaving a geometry that is now equivalent to that of a black hole, instead of a wormhole, in that the surface embedding the ringhole space-time metric (1) always flares inward, being therefore made out of ordinary matter with positive internal energy, which is characterized by a positive temperature.
It is worth remarking, moreover, that the thermal emission process of ringholes can take only place because one can always define a trapping horizon for them, similarly to how we did for the case of wormholes [7]. The interplay between the ringhole angular horizons at $\varphi_{2}=\varphi_{2}^{c}$ and $\varphi_{2}=2 \pi-\varphi_{2}^{2}$ and the trapping horizon (whose existence becomes ultimately responsible also for the thermal emission of wormholes [7]) is depicted in Fig. 3. In fact, this is actually the main argument supporting the Hayward $2+2$ mechanism when adapted for ringholes.

On the other hand, such as was envisaged for the case of black holes [21], and also in the case of ringholes, the thermal radiation can be regarded to be the particle production originating from moving mirrors out of a cosmic vacuum made up of a mixture of dark and phantom energies. Taking the usual mirror trajectory, $x=-t-$ $A \exp (-2 \kappa t)+B$, with $A$ and $B$ given constants, we can get the two limiting situations depicted in Fig. 2 (see Fig. 4). Thus, whereas the limiting space-time (Fig. 2(a)) with $a=b$ is obtained by only emitting phantom radiation at $T<0$, after a time

$$
t^{W} \sim 5 \times 10^{3} \frac{\pi G^{2} M^{3}}{\hbar c^{4}}\left(1-\frac{\varphi_{2}^{c}}{2 \pi}\right),
$$

the limiting space-time (Fig. 2(b)) with $a=-b$, can finally be reached by usual thermal radiation at $T>0$, after a time

$$
t^{B} \sim 5 \times 10^{3} \frac{G^{2} M^{3} \varphi_{2}^{c}}{\hbar c^{4}} .
$$

\section{Thermal Radiation from a Ringhole}

$$
\begin{gathered}
\int_{\text {Surface gravity }} \frac{b_{0}^{2}}{2 b\left(a^{2}+b^{2}-2 a b \cos \varphi_{2}\right)}+2 \pi \sqrt{a^{2}+b^{2}-2 a b \cos \varphi_{2}}\left(\varepsilon-p_{r}\right) \\
\qquad T_{G}=\frac{-\left.\kappa\right|_{b=b_{0}}}{2 \pi} \\
-\varphi_{2}^{c}<\varphi_{2}<\varphi_{2}^{c} \Rightarrow T_{G}>0 \\
2 \pi-\varphi_{2}^{c}>\varphi_{2}>\varphi_{2}^{c} \Rightarrow T_{G}<0
\end{gathered}
$$

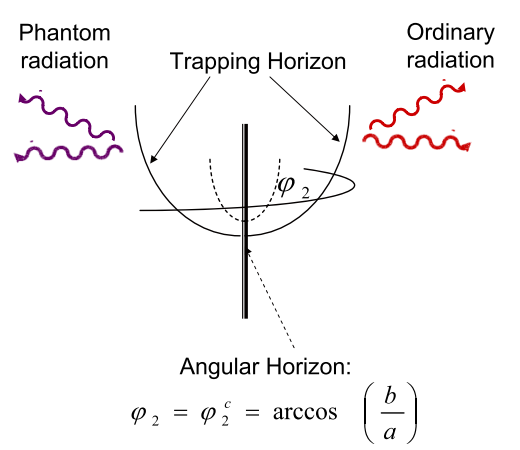

FIG. 3 (color online). The interplay between the angular horizons and the trapping horizons during the thermal emission of ringholes. 

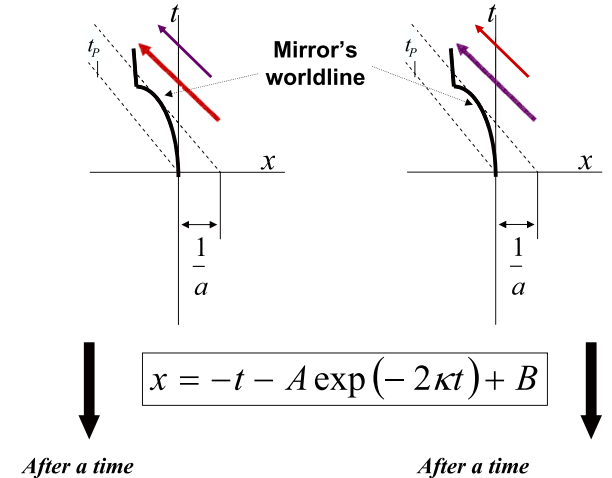

$t^{w} \approx 510^{3}\left(1-\frac{\varphi_{2}^{c}}{2 \pi}\right) \frac{\pi G^{2} M^{3}}{\hbar c^{4}} \quad t^{B} \approx 510^{3} \frac{G^{2} M^{3} \varphi_{2}^{c}}{\hbar c^{4}}$

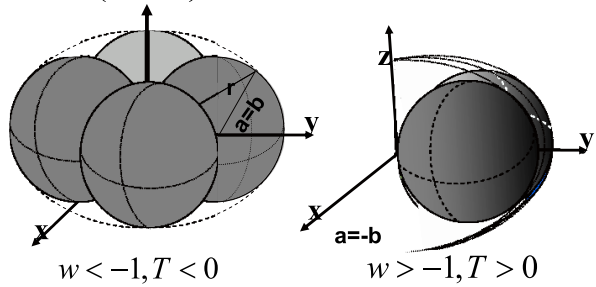

FIG. 4 (color online). Thermal radiation from ringholes envisaged as the radiation from a receding mirror, analogously to how it happens in black holes [21]. Also, in the present case the mode solutions for the receding mirror are the same as the late-time asymptotic modes for a torus of a mixture phantom/dark energy going to form the ringhole.

\section{ACCRETION OF VACUUM ENERGY ONTO RINGHOLES}

We turn now to the problem of the accretion of dark energy onto a ringhole. A treatment similar to those followed for the cases of black holes and wormholes [22] (based on integrating both the conservation laws for the energy-momentum tensor and that for the projection of the energy-momentum on four-velocity), leads us to the following approximate rate equations for the accretion of dark energy in the asymptotic region where $b^{2} \rightarrow a^{2} \rightarrow \infty$,

$$
\dot{M}_{I}=-Q_{I} M^{2}(1+w) \rho,
$$

(with $Q_{I}$ a numerical constant of order unity) for the regime where the embedding surface flares outward with $2 \pi-\varphi_{2}^{c}>\varphi_{2}>\varphi_{2}^{c}$, and

$$
\dot{M}_{I I}=+Q_{I I} M^{2}(1+w) \rho,
$$

(with $Q_{I I}$ another numerical constant of order unity) when the embedding surface flares inward for $-\varphi_{2}^{c}<\varphi_{2}<\varphi_{2}^{c}$, with $\varphi_{2}^{c}=\arccos (b / a)$ in both cases. In deriving Eqs. (8) and (9) dark energy has been modeled as a perfect fluid with a generic equation of state $p=w \rho$, with $p$ and $\rho$ the pressure and energy density, respectively, and $w$ a parameter taking on values that defines the quintessence [17] ( $w>$ $-1)$ and phantom $[18](w<-1)$ regimes,
The above two equations for the mass rate can be rigorously derived from metric (1) when this is expressed in the form [1]

$$
d s^{2}=-d t^{2}+\frac{d r^{2}}{1-\frac{K(b)}{b}}+m^{2} d \varphi_{1}^{2}+b^{2} d \varphi_{2}^{2},
$$

where $K(b)=b_{0}^{2} / b$, and we allow $K(b)$ to actually depend in an arbitrary way on time $t$ as well, so that $K \equiv K(b, t)$ and metric (10) becomes no longer static [23]. Now, if dark energy is taken to be a perfect fluid having an energymomentum tensor $T_{\mu \nu}=(p+\rho) u_{\mu} u_{\nu}+p g_{\mu \nu}$, with $u^{\mu}=d x^{\mu} / d s$ the four-velocity, $u^{\mu} u_{\mu}=-1$, then we can integrate over $r$ [23] the conservation law for the time component of the energy-momentum tensor, $T_{\mu ; \nu}^{\nu}=$ 0 constructed from metric (10), to obtain

$$
\pm u \frac{r^{2}}{M^{2}} \frac{\sqrt{1-\frac{k^{\prime}(r, t)}{r}+u^{2}}}{1-\frac{K^{\prime}(r, t)}{r}}(p+\rho) e^{\int_{\infty}^{r} d r \alpha}=C(t),
$$

where we have introduced a mass factor $\pm M^{-2}$ to provide the right-hand side function $C(t)$ with the dimension of an energy density,

$$
\begin{aligned}
K^{\prime}(r, t) & =\frac{K(b, t)}{\frac{a \cos \varphi_{2}}{r} \pm \sqrt{1-\frac{a^{2} \sin ^{2} \varphi_{2}}{r^{2}}}} \underset{r, a, b \rightarrow \infty}{\rightarrow} \frac{K(r, t)}{2 \cos \varphi_{2}} \\
& \equiv \frac{r_{0}(t)^{2}}{2 r \cos \varphi_{2}} \rightarrow 0,
\end{aligned}
$$

and

$$
\alpha=\frac{\partial_{0} T_{0}^{0}}{T_{0}^{r}}+\frac{\partial_{0} K^{\prime}(r, t)}{2 r\left(1-\frac{K^{\prime}(r, t)}{r}\right)} \frac{T_{0}^{0}-T_{r}^{r}}{T_{0}^{r}},
$$

with $\partial_{0} \equiv \partial / \partial t$.

Now, integrating over $r$ [23] the conservation law for energy-momentum tensor projected on the four-velocity, $u_{\mu} T_{; \nu}^{\mu \nu}=0$, we next get

$$
r^{2} u \frac{e^{\int_{\rho_{\infty}^{\rho}(d \rho /(p(\rho)+\rho))}+\int_{\infty}^{r} d r \beta}}{\sqrt{1-\frac{K^{\prime}(r . t)}{r}}}=A(t),
$$

in which $A(t)$ is a time function having the dimension of a squared mass satisfying that $A(t)=\lim _{a, b \rightarrow \infty} r u^{2}$ does not depend on $r$ but it does on $t$ only through the mass $M \equiv$ $M(t)$, so that $A(t)=A^{\prime} M^{2}$, with $A^{\prime}$ being a dimensionless positive $(u>0)$ constant; we have finally

$$
\begin{aligned}
\beta= & \sqrt{\frac{1-\frac{K^{\prime}(r, t)}{r}+u^{2}}{u^{2}\left(1-\frac{K^{\prime}(r, t)}{r}\right)}}\left[\frac{\partial_{0} \rho}{p+\rho}+\frac{\partial_{0} K^{\prime}(r, t)}{2 r\left(1-\frac{K^{\prime}(r, t)}{r}\right)}\right] \\
& +\frac{\partial_{0}\left(\sqrt{1-\frac{u^{2}}{1-\frac{K^{\prime}(r, t)}{r}}}\right)}{u} .
\end{aligned}
$$


From Eqs. (11) and (13), we now derive

$(p+\rho) \sqrt{\frac{1-\frac{K^{\prime}(r, t)}{r}+u^{2}}{1-\frac{K^{\prime}(r, t)}{r}}} e^{\int_{\rho_{\infty}}^{\rho}(d \rho /(p(\rho)+\rho))+\int_{\infty}^{r} d r(\alpha-\beta)}=B(t)$,

with $B(t)=C(t) / A^{\prime}=p\left[\rho_{\infty}(t)\right]+\rho_{\infty}(t)$. The mass rate due to phantom energy accretion should be finally given by integrating over the toroidal surface area $d S=$ $b m d \varphi_{1} d \varphi_{2}$ the nonzero stress tensor component $T_{0}^{r}$, i.e. $\dot{M} \equiv d M / d t=\int d S T_{0}^{r}$. We then obtain from Eqs. (13) and

$$
\dot{M}=\mp(p+\rho) Q_{ \pm} M^{2} \sqrt{1-\frac{K^{\prime}(r, t)}{r}} e \int_{\infty}^{r} d r \alpha,
$$

with $Q_{ \pm}$being proportional to

$$
\begin{aligned}
& Q_{+} \propto 2 \pi b\left(a \varphi_{2}^{c}+b \sqrt{1-\frac{b^{2}}{a^{2}}}\right) \\
& Q_{-} \propto 2 \pi b\left(a\left(2 \pi-\varphi_{2}^{c}\right)-b \sqrt{1-\frac{b^{2}}{a^{2}}}\right) .
\end{aligned}
$$

The choice of signs in Eq. (16) has been made so that the sign accounts for the $\varphi_{2}$ interval where the embedding surface flares outward like in the wormhole case, and the sign + accounts for the $\varphi_{2}$ interval yielding up an inward flaring of the embedding surface, such as it happens in the case of black holes. Finally, for the accretion of a general perfect fluid dark energy, we recover in the asymptotic region $b^{2} \rightarrow a^{2} \rightarrow r^{2} \rightarrow \infty$ [23] the same expressions (8) and (9), with $Q_{I}, Q_{I I} \propto 4 \pi^{2}$.

Using the expression for the energy density which can be derived by integrating the conservation law for cosmic energy in an accelerating Universe, that is $\rho=$ $\rho_{0} a^{-3(1+w)}$ [24], and a general scale factor for the accelerating Universe given by [25]

$$
\begin{aligned}
a(t) & =T^{2 / 3(1+w)} \\
& =\left[a_{0}^{3(1+w) / 2}+\frac{3}{2}(1+w) C\left(t-t_{0}\right)\right]^{2 / 3(1+w)},
\end{aligned}
$$

with $C=\sqrt{8 \pi G \rho_{0} / 3} \equiv \ell_{P} \sqrt{\rho_{0}}$, one can integrate the above two rate equations. For the quintessence regime where $w>-1$, we can then obtain in the case of the exotic, phantomlike component of the ringhole mass inducing a flaring outward of the embedding surface

$$
M_{I}=\frac{M_{I 0}}{1+\frac{Q_{I} \rho_{0} M_{I 0}(1+w)\left(t-t_{0}\right)}{T(w>-1)}},
$$

in which, and in expressions to follow, $M_{I 0}$ is the initial exotic mass in the ringhole, and in the case of its ordinary energy component that induces a flaring inward of the embedding surface

$$
M_{I I}=\frac{M_{I I 0}}{1-\frac{Q_{I I} \rho_{0} M_{I 00}(1+w)\left(t-t_{0}\right)}{T(w>-1)}},
$$

where, and in expressions to follow, $M_{I I 0}$ is the initial ordinary mass in the ringhole.

In Eqs. (18) and (19) $M_{I}$ and $M_{I I}$ give the mass/energies corresponding to the regions flaring outward and inward, respectively. These mass/energies can be shown to be proportional to the size of the respective spatial regions in the ringhole.

Now, if the Universe is dominated by a phantom energy vacuum [18], then, corresponding to the angular domain $2 \pi-\varphi_{2}^{c}>\varphi_{2}>\varphi_{2}^{c}$, we get

$$
M_{I}=\frac{M_{I 0}}{1-\frac{Q_{I} \rho_{0} M_{I 0}(|w|-1)\left(t-t_{0}\right)}{T(w<-1)}}
$$

and for $-\varphi_{2}^{c}<\varphi_{2}<\varphi_{2}^{c}$,

$$
M_{I I}=\frac{M_{I I 0}}{1+\frac{Q_{I I} \rho_{0} M_{I I 0}(|w|-1)\left(t-t_{0}\right)}{T(w<-1)}} .
$$

It finally follows from an inspection of expressions (18)(21) that if the Universe is dominated by quintessence (phantom) energy with $w>-1(w<-1)$, as time goes on, the ringhole tends to become of the limiting ringhole type B (A) [see Fig. 2] for $a=-b(a=b)$, which were considered to be also the two possible final ringhole configurations, with the smallest sizes, in the case of thermal emission considered in the precedent section.

The first of these limiting configurations takes place whenever the current Universe is filled with a quintessence fluid and the ordinary energy corresponding to the surface which flares inward, $M_{I I}$, diverges and the phantom energy associated with the flaring outward surface tends to the limiting smallest value

$$
M_{I \min }=\frac{M_{I 0}}{1+\frac{Q_{I} M_{I 0}}{Q_{I I} M_{I I 0}}} .
$$

This limiting ringhole with divergent energy $M_{I I}$ and minimal energy $M_{I}$ for $a=-b$ (see Fig. 2(b)) would take place at a time in the future given by

$$
t=t_{0}+\frac{a_{0}^{3(1+w) / 2}}{(1+w)\left(Q_{I I} \rho_{0} M_{I I 0}-\frac{3}{2} C\right)},
$$

provided that the quasiquantum condition $Q_{I I} \sqrt{\rho_{0}} M_{I I 0}>$ $\ell_{P}$ holds, where $\ell_{P}$ is the Planck length.

The second limiting configuration occurs whenever the current Universe is filled with a phantom energy fluid and now it is the phantomlike energy in the ringhole that corresponds to the surface which flares outward, $M_{I}$, which is diverging while the positive, ordinary energy associated with the flaring inward surface tends to another limiting smallest value 


$$
M_{I I \min }=\frac{M_{I I 0}}{1+\frac{Q_{I I} M_{I I 0}}{Q_{I} M_{I 0}}} .
$$

This limiting ringhole with divergent energy $M_{I}$ and minimal energy $M_{I I}$ can be shown to exist for $a=b$ (see Fig. 2(a)), and would take place at a time in the future given by

$$
t=t_{0}+\frac{t_{b r}-t_{0}}{\frac{Q_{l} \rho_{0} M_{l 0}}{C}+\frac{3}{2}}
$$

with $t_{b r}$ the time at which the big rip singularity [8] takes place, that is

$$
t_{b r}=t_{0}+\frac{2 a_{0}^{-3(|w|-1) / 2}}{3(|w|-1) C}
$$

In this case, the size of the outward-flaring region increases in such a way that it becomes able to engulf the Universe itself even before the advent of the big rip singularity. A similar behavior takes places as well in the case of spherically symmetric wormholes, a phenomenon which has been dubbed the big trip [22] and that could ultimately mean the ultimate demise or saving of the Universe itself.

In Ref. [7], it was shown that, at least in some important respects, the dark energy accretion process onto wormholes ought to be the time-reversed version from the wormhole the thermal emission process. One should expect such an equivalence to hold also in the case of ringholes. Thus, it is actually proof of the consistency of the results derived in Sec. III in the case of the ringhole thermal emission that such results give rise as well to limiting situations which are the time-reversed version from those obtained when dark energy is accreted onto ringholes.

We can finally reconfirm a previous conclusion implicitly drawn from the application of the quantum interest conjecture to ringholes and wormholes (see Sec. III). The belief in such a conclusion actually requires two conditions to be fulfilled: First of all, and such as was already suggested in Ref. [7] and mentioned above, the thermal emission and the accretion processes are related to each other in such a way that we can see one as the time-reversed version of the other. If so, then the second condition would dictate that whereas in a vacuum with positive internal energy the Ford-Roman quantum interest conjecture [6] holds, if the vacuum is made out of phantom energy, or any fluid having negative internal energy, then what could be called a quantum altruism conjecture starts holding, implying that a pulse with positive internal energy must always be overcompensated by one with negative internal energy, the difference between the two pulses being in this case proportional to the time elapsed from the emission of the positive internal energy pulse and the emission of the negative-energy pulse (see Fig. 5).

In fact, since the two subsystems, respectively, having positive and negative temperature in a single ringhole can
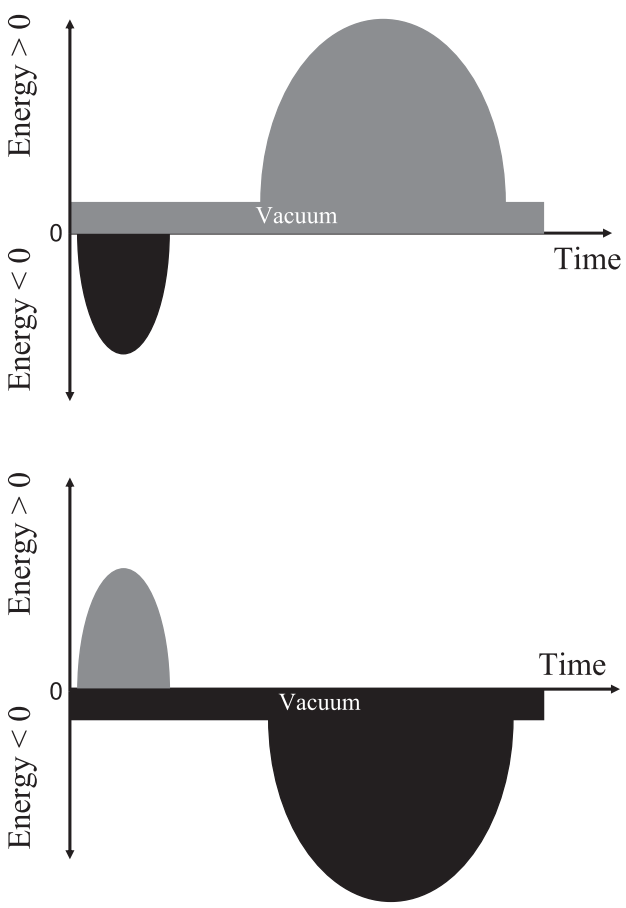

FIG. 5. Upper part: The quantum interest conjecture. Pulses of negative internal energy are allowed by quantum theory in a vacuum with positive internal energy provided the three conditions first introduced by Ford and Roman are fulfilled [6]. Lower part: The quantum altruism conjecture. Pulses with positive internal energy would be permitted by quantum theory in a vacuum with negative internal energy under the following three conditions: (1) The longer the positive pulse lasts, the weaker it must be. (2) A pulse with negative internal energy must follow whose magnitude exceeds that of the initial positive internal energy pulse. (3) The longer the time interval between the two pulses, the larger the negative internal energy pulse must be.

never be in contact, despite that $|T>0|$ is always smaller than $|T<0|$ by virtue of Eqs. (8) and (9), it can be clearly deduced that, on the one hand, if the Universe in which there is an evolving ringhole is dominated by a positive internal energy fluid (i.e. $\rho+p>0$ ), such as dark energy quintessence, the whole ringhole system will be progressively enriched in its positive-temperature component over that with negative temperature as time goes on, in such a way that any emitted pulse with negative internal energy can always be overcompensated by a pulse with positive internal energy, and conversely, if on the other hand, the Universe is dominated by a negative internal energy fluid (i.e. $\rho+p<0$ ), such as the phantom energy, then the whole ringhole system will be progressively enriched in its negative-temperature component over that with positive temperature as time goes on, and therefore any emitted pulse with positive internal energy can always be overcompensated by a pulse with negative internal energy, in both cases the difference between the two pulses increasing as the time elapsed between them becomes longer. 


\section{SUMMARY AND FURTHER COMMENTS ON RINGHOLE THERMODYNAMICS}

The ringholes we are dealing with are generally traversable space-time tunnelings whose throat satisfies the topology of a torus. This paper deals with the thermal properties of ringholes both when they are isolated and when they are immersed in a cosmic vacuum having a given dark energetic content. We have found that a single ringhole possesses a $\varphi_{2}$ - angular region (with $\varphi_{2}$ the angle defined on the circular section with radius $b$ on the torus, (see Fig. 1(a)), characterized by a negative temperature which is not in contact with another angular region with positive temperature from which it is separated by means of well-defined horizons upon which the temperature is always zero. Depending on the values taken on by the set of geometric parameters defining the toroidal throat (Fig. 1(a) ), the fate of a ringhole after thermal emission tends to reach a limiting ringhole structure characterized by either just a negative or a positive temperature. These two limiting ringholes are also the final destiny of the dark energy accretion processes onto ringholes with arbitrary sizes, the difference being that in the latter case the final size of the limiting ringholes tends to be divergent.

On the other hand, according to the known principles of gravitational thermodynamics [26], one-fourth of the surface area of the torus making the throat of the ringhole is expected to provide us with the ringhole entropy, $S_{\mathrm{RH}}$ [26]. Thus, we would have

$$
S_{\mathrm{RH}}=\frac{b}{4 \ell_{P}^{2}} \int_{0}^{2 \pi} d \varphi_{1} \int_{0}^{2 \pi} d \varphi_{2} m=\frac{\pi^{2} a b}{\ell_{P}^{2}} .
$$

We finally notice that even though ringholes do not show an event horizon, they must have a trapping horizon which is nothing but that for spherically symmetric wormholes [7] when generalized to toroidal symmetry. The existence of such a trapping horizon appeared as the ultimate reason why a temperature and an entropy, and actually an entire thermodynamics, are properties pertaining to ringholes, such as it occurs in wormholes [7]. In what follows, I shall tentatively state the familiar laws that one would expect the ringhole thermodynamics should satisfy. Basing on reconciling the laws of thermodynamics for ordinary matter and radiation, black holes [27], wormholes [7], the de Sitter universe [28], and warp drives [29] with the existence of a trapping horizon in the ringhole, we now seek to tentatively formulate the four laws of ringhole thermodynamics as physical properties that we expect the ringholes would have. These laws would be analogous to those of the thermodynamics for ordinary matter and radiation, black holes, wormholes, Unruh vacuum [30], or the de Sitter universe, and may be stated as follows. (i) The surface gravity for a stationary ringhole would no longer be strictly constant, in the present case meaning that $\kappa \equiv \kappa\left(\varphi_{2}\right)$ remains constant along time for every value of the angle $\varphi_{2}$, not that $\kappa$ is kept constant irrespective of the angle $\varphi_{2}$.
This would express a zeroth law. (ii) A first law would then state that

$$
d M=\frac{\kappa}{8 \pi} d A_{\mathrm{RH}}
$$

that is,

$$
\frac{d M}{d \varphi_{2}}=\frac{1}{4} \kappa m, \quad \frac{d M}{d \varphi_{1}}=\frac{1}{4} \kappa a b,
$$

where $\kappa$ is the surface gravity given by Eq. (5), and $M=$ $M_{I}+M_{I I}$. (iii) If one now assumes the weak energy condition to hold, then the inequality

$$
d A_{\mathrm{RH}} \geq 0
$$

would express the second law in case the ringhole is placed in an empty environment. For the most realistic situation in which the ringhole is surrounded by some ordinary material and/or radiation, so as gravitationally compact objects, such as black holes, wormholes, warp drives, or the Unruh vacuum, then this second law had to be generalized to also encompass the entropies of all of these materials and objects [31]. Finally, (iv) the statement that it is not possible to have a ringhole with $\kappa=0$ would amount to the description of a third law.

A comment on the concept of negative phantom temperature is worth including at this stage. In the first consideration made on dark energy thermodynamics, Lima and Alcaniz [32] reached the conclusion that whereas the temperature of a phantom fluid with vanishing chemical potential, $\mu=0$, its entropy should be negative definite so rendering the phantom phase physically meaningless [32] (see also the work by Izquierdo and Pavon [33] and Brevik et al. [34].) Nevertheless, Lima himself together with Pereira [35,36] later found that if we took the chemical potential to be negative, $\mu<0$, then the phantom phase could be characterized by positive values of temperature, entropy, and density. However, it was shown $[19,37]$ that even for $\mu=0$ one can consistently argue that the temperature of a phantom fluid must still be negative definite, with both the entropy and density being positive. When this issue is investigated in a rather general and unified way [38], then all quantities (including a varying equation-ofstate parameter $w(a))$ become well defined and regular for every $w(a)$, with the temperature always negative in the phantom regime, and always positive in the quintessence one. It is also found [38] that the entropy and density are always positive while the chemical potential can be arbitrary. Moreover, at the -1 crossing both the temperature and the chemical potential become strictly zero. The negativity of temperature can only be interpreted in the quantum framework, a domain which is most natural for wormhole [7] and ringhole thermally radiating, and exotic phantom fluid whose energy density tends to diverge as the cosmic scale factor reaches the future singularity. Furthermore, the regular behavior of all quantities at the -1 crossing leads to 
the conclusion that such a crossing should correspond to a smooth crossover, rather than a phase transition.

On the other hand, enclosing a ringhole in a box, having perfectly reflecting walls, which also contains arbitrary amounts of ordinary radiation and phantom radiation [21], and then letting the whole system to quantum mechanically evolve in time, should always produce an increase in entropy, in agreement with the generalized second law [31], no matter whether or not quantum gravity is a time-symmetric theory [39]. However, in order to show that this is really the case, one ought to use an even more careful treatment than that employed when dealing with systems whose components have all positive internal en- ergy and positive temperature [40]. In fact, e.g. it is known [41] that systems that are characterized by a negative temperature are able to only show a finite number of modes available, and this may finally result in a reduction in entropy when positive energy is added onto the system.

\section{ACKNOWLEDGMENTS}

This work was conceived and partly developed in the Estación Ecológica de Biocosmología of Medellín, Spain. The author is indebted to C. Siguienza for useful discussions. This work was supported by the Spanish MICINN under Research Project No. FIS2008-06332/FIS.
[1] P. F. González-Díaz, Phys. Rev. D 54, 6122 (1996).

[2] See the contributions to Les Houches Black Holes, edited by C. DeWitt and B.S. DeWitt (Gordon and Breach Science Publishers, New York, 1972).

[3] M. Visser, Lorentzian Wormholes: From Einstein to Hawking (Springer-Verlag, New York, 1997).

[4] P. F. González-Díaz, arXiv:1001.3778.

[5] R. Gavazzi, T. Treu, L. V.E. Koopmans, A. S. Bolton, L. A. Moustakas, S. Burles, and P.J. Marshall, arXiv:0801.1555.

[6] L.H. Ford and T. A. Roman, Phys. Rev. D 60, 104018 (1999).

[7] P. Martín-Moruno and P. F. González-Díaz, Phys. Rev. D 80, 024007 (2009); Classical Quantum Gravity 26, 215010 (2009).

[8] R. R. Caldwell, Phys. Lett. B 545, 23 (2002); S. M. Carroll, M. Hoffman, and M. Trodden, Phys. Rev. D 68 , 023509 (2003); R. R. Caldwell, M. Kamionkowski, and N. N. Weinberg, Phys. Rev. Lett. 91, 071301 (2003); P. F. González-Díaz, Phys. Lett. B 586, 1 (2004); Phys. Rev. D 69, 063522 (2004)

[9] S. A. Hayward, Phys. Rev. D 49, 6467 (1994); 53, 1938 (1996); 70, 104027 (2004); Classical Quantum Gravity 15, 3147 (1998); Int. J. Mod. Phys. D 8, 373 (1999).

[10] M. Morris and K. S. Thorne, Am. J. Phys. 56, 395 (1988).

[11] P. F. González-Díaz, Phys. Rev. D 68, 084016 (2003); 56, 6293 (1997).

[12] J. P. S. Lemos and V. T. Zanchin, Phys. Rev. D 54, 3840 (1996).

[13] S. L. Shapiro, S. A. Teukolsky, and J. Winicour, Phys. Rev. D 54, 3840 (1996).

[14] S. W. Hawking and G. F. R. Ellis, The Large Scale Structure of Space-Time (Cambridge University Press, Cambridge, UK, 1973).

[15] A. Einstein and N. Rosen, Phys. Rev. 48, 73 (1935).

[16] R. W. Fuller and J.A. Wheeler, Phys. Rev. 128, 919 (1962).

[17] C. Wetterich, Nucl. Phys. B302, 668 (1988); J. C. Jackson and M. Dodgson, Mon. Not. R. Astron. Soc. 297, 923 (1998); J. C. Jackson, Mon. Not. R. Astron. Soc. 296, 619 (1998); R. R. Caldwell, R. Dave, and P. J. Steinhardt, Phys.
Rev. Lett. 80, 1582 (1998); L. Wang and P. J. Steinhardt, Astrophys. J. 508, 483 (1998); R. R. Caldwell and P. J. Steinhardt, Phys. Rev. D 57, 6057 (1998); G. Huey, L. Wang, R. Dave, R. R. Caldwell, and P. J. Steinhardt, Phys. Rev. D 59, 063005 (1999); P. F. González-Díaz, Phys. Rev. D 62, 023513 (2000).

[18] R. R. Caldwell, Phys. Lett. B 545, 23 (2002); R. R. Caldwell, M. Kamionkowski, and N. N. Weinberg, Phys. Rev. Lett. 91, 071301 (2003); P. F. González-Díaz, Phys. Lett. B 586, 1 (2004).

[19] P. F. González-Díaz and C. L. Sigüenza, Nucl. Phys. B697, 363 (2004).

[20] A. ShatskiyAstron. Zh. 84, 99 (2007) [Astronomy Reports 51, 81 (2007)].

[21] P. C. W. Davies and S. A. Fulling, Proc. R. Soc. A 354, 59 (1977).

[22] P. F. González-Díaz, Phys. Rev. Lett. 93, 071301 (2004); P. F. González-Díaz and J. A. Jiménez-Madrid, Phys. Lett. B 596, 16 (2004).

[23] P. F. González-Díaz, Phys. Lett. B 632, 159 (2006).

[24] P.J.E. Peebles, Principles of Physical Cosmology (Princeton University Press, Princeton, NJ, 1976).

[25] P. F. González-Díaz, Phys. Rev. D 68, 084016 (2003).

[26] P. F. González-Díaz, Phys. Rev. D 27, 3042 (1983).

[27] J. M. Bardeen, B. Carter, and S. W. Hawking, Commun. Math. Phys. 31, 161 (1973).

[28] G. W. Gibbons and S. W. Hawking, Phys. Rev. D 15, 2738 (1977).

[29] P. F. González-Díaz, Phys. Lett. B 653, 129 (2007).

[30] W. G. Unruh, Phys. Rev. D 14, 870 (1976).

[31] J. D. Bekenstein, Phys. Rev. D 9, 3292 (1974).

[32] J.A.S. Lima and J.S. Alcaniz, Phys. Lett. B 600, 191 (2004).

[33] G. Izquierdo and D. Pavón, Phys. Lett. B 633, 420 (2006).

[34] I. Brevik, S. Nojiri, S. D. Odintsov, and L. Vanzo, Phys. Rev. D 70, 043520 (2004).

[35] J.A. S. Lima and S. H. Pereira, Phys. Rev. D 78, 083504 (2008).

[36] S. H. Pereira and J.A. S. Lima, Phys. Lett. B 669, 266 (2008).

[37] Y. S. Myung, Phys. Lett. B 671, 216 (2009). 
[38] E. N. Saridakis, P. F. González-Díaz, and C. L. Sigüenza, Classical Quantum Gravity 26, 165003 (2009).

[39] R. Penrose, Time-Asymmetry and Quantum Gravity, in: Quantum Gravity 2: A Second Oxford Symposium edited by C. J. Isham, R. Penrose, and D. W. Sciama (Clarendon
Press, Oxford, UK, 1981).

[40] S. W. Hawking, Phys. Rev. D 13, 191 (1976).

[41] E. M. Purcell and R. V. Pound, Phys. Rev. 81, 279 (1951); N. F. Ramsey, Phys. Rev. 103, 20 (1956). 